\title{
O REFORÇAMENTO DA COOPERAÇÃO ENTRE CRIANÇAS ${ }^{1}$
}

\author{
THE REINFORCEMENT OF COOPERATION BETWEEN CHILDREN
}

\author{
NATHAN H. AZRIN \\ HARVARD UNIVERSITY, EUA \\ OGDEN R. LINDSLEY \\ HaRVARD Medical School, EUA
}

O presente texto é uma tradução do original: Azrin, N. H. \& Lindsley, O. R. (1956). The reinforcement of cooperation between children. Journal of Abnormal and Social Psychology, 52, 100-102. O texto encontra-se em domínio público, dispensando autorização. Algumas expressões foram traduzidas literalmente para preservar a linguagem utilizada na época, como "psicótico" e "organismos inferiores".

A tradução é de Alceu Regaço, Letícia dos Santos e Lucas Couto de Carvalho, do INCT-ECCE - Laboratório de Psicologia da Aprendizagem, Departamento de Psicologia, UFSCar. Os primeiros tradutores são bolsistas de iniciação científica (FAPESP: Processo 2016/17.067-9 e Processo 2016/17.069-1). O terceiro é bolsista de pós-doutorado (FAPESP: \#2017/13840-8). Os tradutores agradecem à professora Deisy das Graças de Souza e à Aurea de Fatima Regaço pelos comentários e ajuda na realização da tradução.

1. Nota original: Esse artigo foi apresentado no encontro da Eastern Psychological Association em 10 de Abril de 1954 na cidade de Nova Iorque.

DOI. 10.18542/rebac.v14i1.7162 
A maioria dos métodos utilizados para as análises experimentais e do desenvolvimento do comportamento de cooperação entre humanos requerem instruções específicas sobre a relação de cooperação entre as respostas individuais. Peters e Murphree (1954) desenvolveram um dos métodos mais recentes sobre o tema. Skinner (1953) sugeriu e demonstrou, usando organismos inferiores (Skinner, 1952), que a cooperação entre indivíduos pode ser produzida, mantida e eliminada simplesmente manipulando a contingência entre o estímulo reforçador e a resposta cooperativa.

As vantagens de se eliminar as instruções no processo de cooperação são (i) a aquisição inicial da cooperação pode ser investigada, (ii) sujeitos com dificuldades de aprendizagem sob contingências de modelação e instrução (i.e., bebês, certas classes de psicóticos e organismos inferiores) podem ser estudados e (iii) ausência de problemas envolvendo efeitos das instruções sobre o comportamento dos sujeitos.

Algumas das vantagens mais gerais das técnicas de condicionamento operante são (i) obtém-se um registro mais contínuo do processo cooperativo, (ii) variáveis ambientais intervenientes são minimizadas e (iii) um tempo relativamente longo de observações experimentais é possibilitado.

\section{PROBLEMA}

A cooperação entre crianças pode ser desenvolvida, mantida e eliminada somente pela apresentação ou não de um único estímulo reforçador, acessível para cada membro do grupo cooperativo, seguindo cada resposta cooperativa?

\section{TIMES COOPERATIVOS}

Vinte crianças, entre sete e 12 anos de idade, formaram dez times cooperativos. As duplas foram formadas de acordo com idade e sexo. Sete times foram formados por meninos e três, por meninas ${ }^{1}$. A seleção foi feita por uma solicitação "Quem quer jogar um jogo?". Os dois primeiros voluntários do mesmo sexo e idade formavam uma dupla. As idades informadas pelas crianças eram confirmadas por meio dos registros do centro comunitário. Nenhuma informação sobre o jogo foi fornecida durante a seleção. Nenhum time foi recusado.

\section{RESPOSTA COOPERATIVA}

A cooperação foi assegurada por meio do planejamento de um aparato (Figura 1) que (i) não podia ser operado por um indivíduo sozinho (assegurando comportamento de grupo) e (ii) exigia que um indivíduo respondesse ao comportamento do outro indivíduo para que se produzisse reforçadores (garantindo a cooperação).

\footnotetext{
${ }^{1}$ Nota original: Gostaríamos de agradecer a Herriet Tubman House e a South Bay Union de Boston, Mass., por fornecerem as instalações e oportunidade de convidarmos as crianças para participarem do experimento.
}

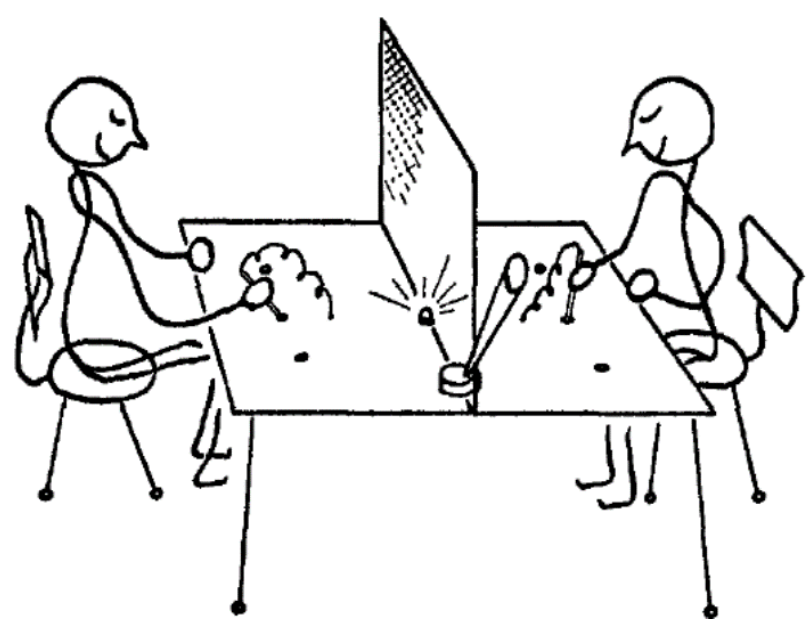

Figura 1. Equipamento usado para o reforçamento da cooperação entre crianças.

\section{PROCEDIMENTO}

As duas crianças de cada time sentavam-se em lados opostos de uma mesa, que continha três buracos e uma caneta, em frente de cada uma (ver Figura 1). Uma tela de arame fixada no centro da mesa impedia que uma criança manipulasse a caneta da outra criança, que estava do outro lado da mesa.

As seguintes instruções foram fornecidas: "Isso é um jogo. Você pode brincar da maneira que quiser ou fazer qualquer outra coisa que preferir. $\mathrm{O}$ jogo funciona da seguinte maneira: coloque as duas canetas dentro de todos os três buracos" (essa instrução foi repetida até que ambas as canetas fossem inseridas nos três buracos). "Enquanto vocês estiverem nesta sala, algumas dessas jujubas" (nesse momento, o experimentador exibia as jujubas em suas mãos) "serão colocadas dentro deste copo. Vocês poderão comê-las aqui, ou levá-las para suas casas." As instruções eram, então, repetidas sem que houvessem respostas para quaisquer perguntas e, em seguida, o experimentador disse: "Vou me retirar da sala agora; você pode brincar do que preferir enquanto eu estiver ausente". Após a instrução, o experimentador saiu da sala até o término da sessão experimental.

$\mathrm{Se}$ as canetas fossem posicionadas nos buracos opostos um do outro dentro de 0,04 segundo (resposta cooperativa), uma luz vermelha acendia na mesa (estímulo reforçador condicionado) e uma única jujuba (estímulo reforçador) caía em um recipiente acessível às duas crianças $^{2}$. As respostas cooperativas foram registadas em

\footnotetext{
${ }^{2}$ Nota original: Skinner (1952) apresentou dois estímulos reforçadores (um para cada pombo) seguido de cada resposta cooperativa.
} 
contadores e o registro das respostas acumuladas foi feito em uma sala adjacente.

\section{Design experimental}

Cada dupla participou de uma única sessão experimental dividida em três períodos diferentes e contínuos, sem interrupção experimental:

Primeiro período de reforçamento. Nesta condição, toda resposta cooperativa foi reforçada por um período mínimo de 15 minutos. Caso a taxa de respostas não estivesse estável ao final do período de 15 minutos, a condição permanecia em vigor até que nenhuma mudança na taxa de respostas cooperativas fosse notada por pelo menos cinco minutos consecutivos.

Período de extinção. As respostas cooperativas não eram reforçadas por um período mínimo de 15 minutos e até que houvesse uma taxa estável de respostas durante, no mínimo, cinco minutos da condição.

Segundo período de reforçamento. As respostas cooperativas voltaram a ser reforçadas até que houvesse uma taxa estável de respostas durante, no mínimo, três minutos. Isto foi feito para verificar se uma diminuição na taxa no último período de extinção ocorreria devido à extinção, saciedade ou fadiga.

\section{RESULTADOS}

Todas as duplas aprenderam a cooperar sem o auxílio de instruções especificas nos primeiros dez minutos de sessão. Observações das sessões por meio de um espelho de observação unilateral revelaram que a maioria dos times cooperativos desenvolveram e mantiveram uma relação de líder-seguidor. Oito duplas quase que prontamente dividiram as balas de alguma forma. Para duas duplas, um dos membros pegou todas as balas fornecidas, até que o outro se recusou a cooperar. Após um acordo verbal ter sido proposto entre os dois membros de cada dupla, eles voltaram a cooperar e a dividir as balas. A maioria das vocalizações ocorreram durante o período inicial de aquisição e durante o período de extinção das respostas cooperativas. As vocalizações foram correlacionadas aos momentos de maior variabilidade na taxa durante os períodos citados (ver abaixo).

A Figura 2 mostra o registro cumulativo das respostas cooperativas das três duplas com o maior, a dupla que representava a mediana, e o menor número de respostas cooperativas durante a sessão experimental. As curvas do gráfico mostram uma grande diferença na taxa de aquisição de cooperação. Uma dupla levou quase 10 minutos para adquirir uma taxa alta de respostas cooperativas. Taxas estáveis de cooperação podem ser observadas durante os últimos minutos do primeiro período de reforçamento. O declínio gradual da cooperação durante a extinção, de forma mais significativa do que o declínio imediato, sugere um padrão da extinção do comportamento cooperativo similar ao que é encontrado em curvas de extinção de respostas individuais. Em todos os casos, a variação da taxa foi maior durante a extinção do que durante o reforçamento. Skinner (1953) encontrou esse aumento da variabilidade na taxa de respostas durante a extinção em experimentos com organismos inferiores e o descreveu como comportamento emocional (p. 69). A alta taxa de respostas após o primeiro reforço do segundo período de reforçamento mostra que a reaquisição é quase imediata.

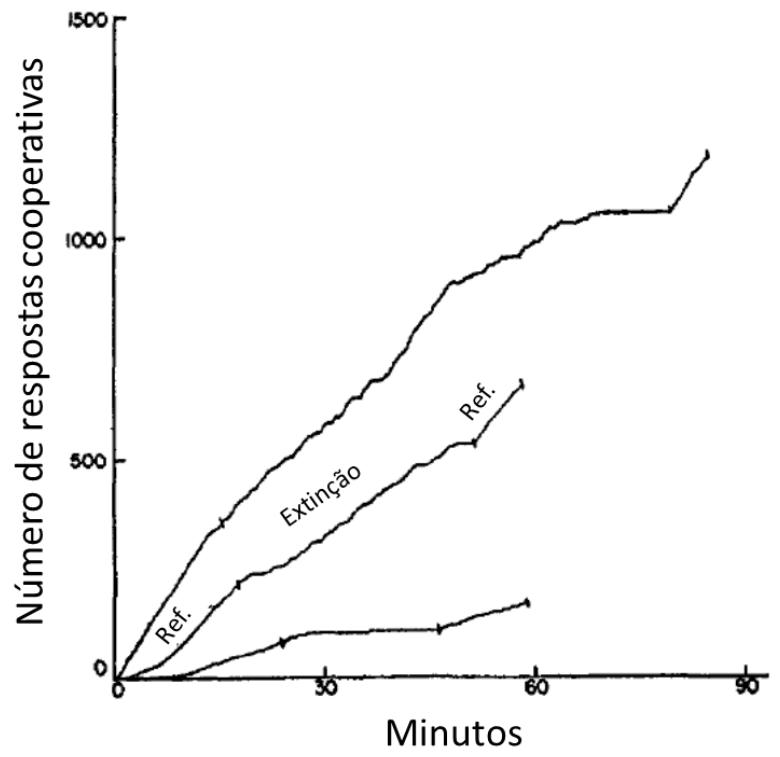

Figura 2. Registros cumulativos de respostas para duplas com o maior, o mediano, e o menor número de respostas cooperativas.

A Tabela 1 mostra a análise estatística dos resultados. São apresentadas a mediana e o maior e menor valor do número de respostas cooperativas por minuto para todas as dez duplas durante os períodos críticos do experimento. $\mathrm{O}$ número de respostas cooperativas por minuto durante os primeiros três minutos do primeiro período de reforçamento, foi significativamente menor que a taxa durante os últimos três minutos do primeiro período de reforçamento $(p<0,02){ }^{3}$ Isto mostra que a taxa de cooperação foi significativamente menor durante a aquisição inicial do que durante a manutenção da cooperação. $\mathrm{O}$ número de respostas cooperativas por minutos durante os últimos três minutos de extinção foi significativamente menor do que a taxa durante os últimos três minutos do primeiro período de reforçamento ( $p<$ $0,001)$. Isto mostra que a remoção do reforçamento durante a extinção diminuiu significativamente a taxa de cooperação entre essas crianças.

\footnotetext{
${ }^{3}$ Nota original: $\mathrm{O}$ teste não paramétrico $\mathrm{T}$ de Wilcoxon para amostras emparelhadas foi usado em todos os tratamentos estatísticos.
} 
Tabela 1.

Mediana e Valores Mínimo e Máximo do Número de Respostas Cooperativas por Minuto Durante os Períodos Experimentais Críticos.

\begin{tabular}{|c|c|c|c|c|}
\hline \multirow[b]{2}{*}{ N 10} & \multicolumn{4}{|c|}{ Número de respostas cooperativas por minuto } \\
\hline & $\begin{array}{l}1 \text { ㅇs três } \\
\text { min. do } 1 \text { o } \\
\text { período de } \\
\text { ref. }\end{array}$ & $\begin{array}{l}\text { Últimos três } \\
\text { min. do } 1 \text { o } \\
\text { período de } \\
\text { ref. }\end{array}$ & $\begin{array}{c}\text { Últimos três } \\
\text { min. do } \\
\text { período de } \\
\text { extinção. }\end{array}$ & $\begin{array}{c}\text { Últimos três } \\
\text { min. do } 2 \underline{0} \\
\text { período de } \\
\text { ref. }\end{array}$ \\
\hline Mediana & 5,5 & 17,5 & 1,5 & 17,5 \\
\hline Variação & $1-26$ & $6-26$ & $0-7$ & $6-27$ \\
\hline
\end{tabular}

O número de respostas cooperativas por minuto durante os últimos três minutos do segundo período de reforçamento foi significativamente maior que a taxa durante os três últimos minutos do período de extinção $(p<0,001)$. Isto mostra que a taxa de cooperação aumentou significativamente durante o segundo período de reforçamento e que a queda da taxa, durante a extinção, está mais relacionada à ausência dos estímulos reforçadores, do que à saciedade ou fadiga. As taxas de cooperação durante o segundo período de reforçamento e os últimos três minutos do primeiro período de reforçamento não foram significativamente diferentes e também mostram que a taxa de respostas foi quase imediatamente reestabelecida ao valor do período pré-extinção na segunda apresentação do estímulo reforçador. A taxa de respostas cooperativas durante os primeiros três minutos do segundo período de reforçamento foi significativamente maior do que durante os três primeiros minutos do primeiro período de reforçamento $(p<0,02)$. Isto demonstra, novamente, que a reaquisição da cooperação não foi gradual, como foi sua aquisição inicial, mas ocorreu quase imediatamente.

\section{CONCLUSÕES}

Técnicas de condicionamento operante podem ser usadas para desenvolver, manter e eliminar cooperação entre crianças sem o uso de instruções específicas relativas à cooperação. A taxa de respostas cooperativas muda como uma função da apresentação de um único reforçador, de forma muito semelhante ao modo como ocorre para respostas individuais. No reforçamento de respostas cooperativas, um estímulo reforçador não precisa ser entregue para cada membro do time cooperativo após cada resposta cooperativa. A apresentação de um único estímulo reforçador, disponível para cada membro do grupo, é suficiente para aumentar a taxa de cooperação. A resposta cooperativa aumenta gradualmente em frequência quando reforçada e decresce em sua frequência quando não é mais reforçada (extinção). Respostas cooperativas são mantidas em uma taxa estável durante o reforçamento, mas ocorrem em explosões esporádicas durante a extinção. O reforçamento, após a extinção, resulta em uma recuperação quase imediata da taxa de cooperação para seus valores anteriores à extinção.

\section{REFERÊNCIAS}

Peters, H. N., \& Murphree, O. D. (1954). A cooperative multiple-choice apparatus. Science, 119, 189-191.

Skinner, B. F. (1953). Science and human behavior. New York, NY: Macmillan.

Skinner, B. F. (1952). Classroom demonstration. Comunicação pessoal.

Wilcoxon, F. (1949). Some rapid approximate statistical procedures. New York, NY: American Cyanamid.

\section{JUSTIFICATIVA DA TRADUÇÃO}

Tendo em vista o crescente número de artigos que tratam sobre o tema de comportamento social, seja a partir de estudos experimentais ou conceituais (Carrara \& Zilio, 2015; Glenn, Malott, Andery, Benvenuti, Houmanfar, Sandaker, Todorov, Tourinho \& Vasconcelos, 2016; Tan \& Hackenberg, 2016; Velasco, Benvenuti, Sampaio, \& Tomanari, 2017;), torna-se importante recuperar estudos anteriores que estudaram o tema, mesmo antes da conceitualização do terceiro nível de seleção do comportamento (Skinner, 1981). O artigo The reinforcement of cooperation between children de Azrin e Lindsley (1956) foi um dos primeiros estudos a serem realizados com o objetivo de estudar o comportamento combinado de dois sujeitos e sua tradução para a língua portuguesa pode contribuir para enriquecer as discussões realizadas nesse campo de pesquisa.

Azrin, N. H. \& Lindsley, O. R. (1956). The reinforcement of cooperation between children. Journal of Abnormal and Social Psychology, 52, 100-102

Carrara, K., \& Zilio, D. (2015). Análise comportamental da cultura: contingência ou metacontingência como unidade de análise. Revista Brasileira de Análise do Comportamento, 11(2), 135-146.

Glenn, S. S., Mallot, M. E., Andery, M. A., Benvenuti, M. F., Houmanfar, R. A., Sandaker, I. ... \& Vasconcelos, L. (2016). Toward consistent terminology in a behavioristic approach to cultural analysis. Behavior and Social Issues, 25, 11-27.

Skinner, B. F. (1981). Selection by consequences. Science, 213, 501-504.

Tan, L. \& Hackenberg, T. D. (2016). Functional analysis of mutual behavior in laboratory rats (Rattus norvegicus). Journal of Comparative Psychology, 130(1), 13-23.

Velasco, S. M., Bevenuti, M. F., Sampaio, A. S., \& Tomanari, G. Y. (2017). Cooperation and metacontingency in pigeons. Psychological Record, 67(4), 537-545. 